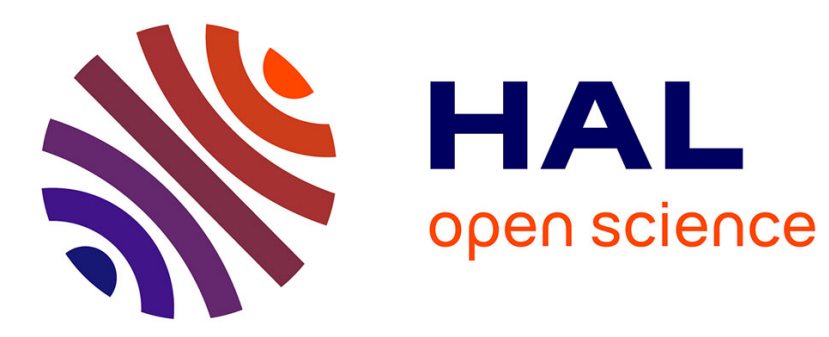

\title{
La corruption du peuple
}

Thierry Ménissier

\section{To cite this version:}

Thierry Ménissier. La corruption du peuple. La Pensée, 2021, pp.27-38. 10.3917/lp.406.0027 . halshs-03556386

\section{HAL Id: halshs-03556386 \\ https://shs.hal.science/halshs-03556386}

Submitted on 4 Feb 2022

HAL is a multi-disciplinary open access archive for the deposit and dissemination of scientific research documents, whether they are published or not. The documents may come from teaching and research institutions in France or abroad, or from public or private research centers.
L'archive ouverte pluridisciplinaire HAL, est destinée au dépôt et à la diffusion de documents scientifiques de niveau recherche, publiés ou non, émanant des établissements d'enseignement et de recherche français ou étrangers, des laboratoires publics ou privés. 


\section{La corruption du peuple}

Thierry Ménissier

Preprint de : Ménissier, T., «La corruption du peuple », La Pensée, 2021/2, n406, numéro spécial «Machiavel, le peuple, la politique, l’expérience », p. 27-38.

\section{Résumé}

S'il peut sembler étrange voire déplacé d'évoquer la corruption du peuple chez un auteur inscrit dans le courant républicain, cette thématique apparaît tout à fait fondamentale pour l'œuvre de Machiavel, attentive à scruter ce qui, dans l'expérience des Anciens, permettrait de fortifier les républiques des Modernes et aurait permis de sauver celle dite du Grand Conseil, le régime qu'il a servi durant quatorze ans. La notion de corruption du peuple s'avère toutefois complexe et difficile à qualifier compte tenu des particularités de la version machiavélienne du républicanisme.

\section{Mots-clés :}

Machiavel, République, corruption, peuple, citoyenneté, bien commun.

\section{Abstract : The Corruption of the People}

If it may seem strange or even out of place to evoke the corruption of the People in an author registered in the republican current, this theme appears quite fundamental for Machiavelli's work, careful to scrutinize what, in the experience of the Ancients, would allow to fortify the republics of the Moderns and would have allowed to save the one called the Great Council, the regime he served during fourteen years. The notion of corruption of the People proves however to be complex and difficult to qualify taking into account the particularities of the Machiavellian version of republicanism.

\section{Keywords :}

Machiavelli, Republic, corruption, People, citizenship, common good. 
« Mais c'est surtout chose très merveilleuse de considérer la grandeur à laquelle parvint Rome après s'être libérée de ses rois. La raison est facile à comprendre, car ce n'est pas le bien des particuliers, mais le bien commun qui rend les villes grandes [non il bene particulare ma il bene comune è quello che fa grandi le città]. Et il ne fait pas de doute que ce bien commun n'est recherché que dans les républiques $»^{1}$.

Lorsqu'on évoque la notion de corruption, c'est toujours en regard d'une volonté de normativité : la conduite ou le comportement désignés comme corrompus le sont toujours par rapport à une norme capable de fixer la rectitude, et souvent en fonction de la volonté de qualifier et de dénoncer des conduites répréhensibles ${ }^{2}$. Or, à lire Machiavel, on pourrait spontanément estimer qu'on se trouverait bien en peine si l'on voulait évoquer, pour son propos, une quelconque intention normative : d'un style souvent sobrement (sinon sèchement) descriptif, le Florentin adopte dans ses œuvres une perspective tour à tour analytique comme le serait un rapport sur la nature du pouvoir (dans Le Prince), comparatiste (en confrontant les Anciens et les Modernes dans le but de louer la force des Républiques des premiers avec les Discours sur la première décade de Tite-Live), enfin historique (en répondant à la commande des Médicis avec l'Histoire de Florence). La notion de corruption pourrait même sembler littéralement désamorcée par un auteur qui semble toujours situer l'exercice du pouvoir «pardelà bien et mal », décourageant par avance la recherche des mœurs du bon gouvernement que l'humanisme civique, en héritier revendiqué de la pensée éthique et politique gréco-latine, avait développée à Florence durant le Quattrocento.

$\mathrm{Si}$, chez Machiavel, de telles complexités surgissent quand on évoque la notion de corruption, les choses semblent plus évidentes concernant celle de peuple : en tant que praticien de l'arte dello stato dans le contexte tendu de ses années d'activité professionnelle, le Secrétaire florentin s'est personnellement engagé en faveur du parti républicain, et, une fois limogé après le retour aux affaires des Médicis et devenu auteur de pensée politique, s'est affilié de manière indiscutable à la tradition de pensée «civile». Ce qui signifie que la notion de peuple est appelée à jouer un rôle majeur : pour un auteur de conviction républicaine, la notion de peuple renvoie au sujet politique collectif de référence, pouvant valoir comme critère de légitimité de la bonne politeia, tant pour penser le régime constitutionnel que pour bâtir les conditions de la socialité de type républicain.

Il faut alors ajouter que, l'auteur des Discours sur la première décade de Tite-Live passant souvent pour le «bon Machiavel », ou même pour le « Machiavel bon », à savoir, celui qui rachète l'auteur «mauvais » du Prince (ainsi que, de la Renaissance jusqu'à Leo Strauss, la tradition de l'anti-machiavélisme s'est évertuée à qualifier le Florentin), évoquer la thématique de la corruption du peuple ne manquera pas d'apparaître comme quelque chose de

1 Machiavel, Discours sur la première décade de Tite-Live, II, 2, § 1. Pour toutes nos références à cette œuvre, nous renvoyons à la traduction par A. Fontana et X. Tabet (Paris, Gallimard, 2004), et à son système de référence qui permet de préciser les paragraphes du texte machiavélien.

2 Nous nous permettons de renvoyer à notre interprétation du phénomène de la corruption : Thierry Ménissier, Philosophie de la corruption, Paris, Hermann, 2018. 
profanateur. Et cela, bien qu'il n'existe pas dans l'œuvre de Machiavel une définition précise, univoque et rigoureuse de la notion de peuple, et rien qui ressemble à un concept philosophique du peuple ou même de la puissance populaire. Le Florentin évoque fréquemment « le peuple de Rome " en faisant souvent référence à l'histoire du Senatus populusque romanus (SPQR), à savoir, au sein de la société oligarchique romaine, à la communauté des citoyens libres capables de se représenter au Sénat. Par analogie, lorsqu'il évoque «le peuple florentin », il envisage moins la force sociale populaire que l'ensemble des citoyens « bourgeois » membres des arti, ces corporations d'arts et métiers qui bénéficiaient de capacité d'expression au sein des Conseils de la République du Grand Conseil. Cette approche s'inscrit elle-même dans la filiation lointaine de la pensée d'Aristote, qui définissait la politique en fonction de la notion de participation, et différenciait les régimes en fonction de la qualité de l'engagement des citoyens ${ }^{3}$

De plus, si l'affiliation de Machiavel au républicanisme est indiscutable, pour autant ce n'est pas quelque chose d'univoque, puisque la version machiavélienne de ce courant de pensée politique apparait « hétérodoxe » à plusieurs égards, notamment en ce qu'il met l'accent sur la fécondité de certains désordres $\left(\right.$ tumulti) ${ }^{4}$ tandis que la vulgate républicaine, autre héritage lointain d'Aristote, se définit plutôt comme favorable au consensus supposé naturel à la communauté civique ${ }^{5}$. Réfléchir aux «manières d'être et de faire » (en termes machiavéliens, $i$ modi) du peuple, c'est réfléchir à la bizarrerie du sujet politique collectif de référence tant pour un régime constitutionnel que pour une socialité et une humanité républicaines.

Évoquer la thématique de la corruption du peuple chez Machiavel revient donc à entrer dans son œuvre par une porte peu aisée à ouvrir. La difficulté se trouve accrue par le fait que cette œuvre est elle-même rédigée (et non publiée du vivant de l'auteur) comme un ensemble de textes nominalement adressés (donc non publics, voire conçus en fonction d'une diffusion strictement restreinte), ce qui la constitue comme un labyrinthe très stimulant pour qui veut penser la politique, cet autre labyrinthe. Mais c'est également une porte qui ouvre sur des perspectives fort stimulantes. Par exemple, que nous dit donc la thématique de la corruption du peuple, pour notre époque où des expressions populaires s'opposent de manière apparemment spontanée à la représentation nationale (dans le cas français des «Gilets jaunes »), et alors qu'un régime démocratique installé depuis longtemps, et qui fut même considéré par de très bons esprits comme un modèle pour toutes les républiques modernes ${ }^{6}$, vient peut-être de connaître un coup d'État populiste manqué (par référence aux événements de l'intrusion de la foule au Capitole à Washington DC, USA, début janvier 2021) ?

\section{La corruption selon Machiavel : une thématique « située » à plusieurs niveaux}

3 Voir Aristote, Politique, III, 1, 1274 b sq. : la cité se définit par l'existence des citoyens, qui se reconnaissent eux-mêmes par le fait qu'ils participent à tour de rôle à l'action publique.

4 Voir Discours, I, 4 : «Que la désunion entre la plèbe et le sénat romain rendit libre et puissante cette république ». 5 Voir Aristote, Politique, III, 9, 1281 a 1 sq. et VII, 4, 1326 b 7 sq. : la cité est une communauté menant une vie autarcique et heureuse.

6 Voir Hannah Arendt, De la Révolution, trad. M. Berrane et J.-F. Hel-Guedj in L'Humaine condition, Paris, Gallimard, 2012. 
Il convient d'abord de considérer que la pensée machiavélienne se comprend en fonction de deux ancrages, le premier dans sa propre actualité, le second en fonction de la référence intellectuelle qu'elle s'est choisie.

\subsection{Le Secrétaire florentin au service d'une république faible :}

La pensée politique de Machiavel, composée «post res perditas », s'ancre dans l'expérience de la République du Grand Conseil (Consiglio Maggiore) qu'il a servie de 1498 à 1512. Ce qui signifie, plus précisément, que Machiavel a officié dans un régime qu'il qualifie lui-même rétrospectivement de faible. Cette faiblesse tient à plusieurs ordres de faits : un modèle constitutionnel fragile ou mal adapté à la « qualité des temps », d'une part, et un manque de virtù de la part des responsables politiques, principalement en ce qui concerne le pouvoir exécutif (Piero Soderini).

Ces deux aspects constituent déjà, tels qu'ils sont analysés dans l'œuvre machiavélienne, une approche du thème de la corruption du peuple. Cette approche coordonne une dimension que nous pourrions nommer constitutionaliste (celle qui réfléchit aux ordini de la République) et une autre tournée vers la psycho-physiologie des tempéraments politiques (celle qui s'attache aux modi de ses acteurs).

La fragilité ou l'inadaptation du modèle constitutionnel florentin provenait de sa complexité et du fait que l'interaction des différents Conseils, servis par les deux Chancelleries, favorisait de mauvais désordres (tumulti) au lieu de ceux qui auraient été favorables à la liberté de la cité. L'un et l'autre entravaient de plus la rapidité d'action et même la latitude du pouvoir exécutif, en des temps où certaines affaires urgentes rendaient nécessaires la promptitude et la résolution. Conjonction fâcheuse, et même fatale au vu de l'effondrement de 1512, ce que le Secrétaire estime être le manque de virtù des responsables: tout particulièrement, Piero Soderini, «gonfalonier à vie » à partir de 1502 et à ce titre chef de l'exécutif, souffrait selon Machiavel d'un double défaut pour exercer sa fonction comme les circonstances l'exigeaient. À son manque de discernement intellectuel s'ajoutait en effet une préjudiciable mansuétude dans les républiques menacées, une des formes de la corruption réside dans les vertus morales dont font preuve ses propres leaders, si tant est que trop d' "humanité » et de "patience » (vertus explicitement attribuées à Soderini) peuvent, en fonction de certaine qualità dei tempi, devenir des vices, très dangereux pour ceux qu'on encadre de par les responsabilités qu'on exerce $^{7}$. Pour le Secrétaire, la doctrine du salut de la patrie conditionne de manière indiscutable les critères de l'éthique publique : "là où il est tout à fait question de décider du salut de la patrie, il ne doit y avoir aucune considération de ce qui est juste ou injuste, compatissant ou cruel, louable ou ignominieux ; au contraire, laissant de côté tout autre égard, il faut suivre entièrement ce parti qui lui sauve sa vie et préserve sa liberté ${ }^{8}$.

\section{2 Un double paradigme hérité de l'Antiquité :}

Tenu éloigné des affaires à partir de 1512, Machiavel s'est tourné vers les écrivains gréco-latins en se plongeant notamment dans la lecture de Tite-Live, historien contemporain de

7 Voir Discours, III, 9, § 3. Machiavel évoque l'action de Soderini en deux autres passages intéressants des Discours : I, 7, 4 et I, 52, 2.

8 Discours, III, 41, 1. 
la montée du principat d'Auguste, dont l'œuvre monumentale vise à montrer la force de la République romaine, menacée et qui connaissait ses derniers jours avant l'apparition de l'Empire au terme de la guerre civile. Dans les Discours sur la première décade de Tite-Live, appel est fait par le Secrétaire florentin à la puissante narration annalistique livienne dans un double but : d'une part, sur le plan de la pensée politique ancrée dans l'actualité récente, évaluer les péripéties vécues par la République du Grand Conseil à l'aune des épisodes fameux et des moments de gloire de la République romaine tels que Tite-Live lui-même les a observés ; de l'autre, mais simultanément, en adoptant du recul par rapport à cette actualité, réfléchir de manière plus générale aux institutions républicaines, aux mœurs qu'elles encadrent et qui les nourrissent, aux situations rencontrées et aux types de tempéraments qui s'y révèlent.

En empruntant aux traditions naturalistes païennes alors en plein revivalisme dans le contexte de l'Italie de la Renaissance, Machiavel écrit qu'en tant que « corps mixtes », toutes les républiques naissent, croissent puis meurent, et également que leur durée de vie dépend de la capacité qu'elles ont, de par leur constitution (au sens d'abord physique et ensuite politique du terme), à être «ramenées à leur commencement ${ }^{9}$. Explicitement évoquée dans cette perspective, la notion de corruption, il convient de le souligner, se trouve liée à une dimension physique, qui apparaît double : elle désigne, sur le plan cosmologique, l'usure des corps individuels et collectifs, et dans l'ordre physico-médical, l'altération qui survient sous la forme de dysfonctionnements pathologiques, et se voit alors pensée d'après la médecine hippocraticogalénique des « humeurs » (umori) en excès ou en défaut.

\section{Une pensée politique de la corruption}

Ces éléments rappelés, il est possible d'entrer dans le détail des arguments exposé par le Florentin. Ils se comprennent à la lumière d'une observation initiale : tandis que, sous l'influence de la pensée libérale, une conception de type juridique domine aujourd'hui la définition de la notion de corruption, Machiavel développe pour sa part, dans une perspective très différente et les éléments de contexte évoqués, une conception de type politique, à l'instar d'ailleurs des penseurs républicains dans leur ensemble ${ }^{10}$.

\subsection{Des mæurs civiques aisément corrompues même en république}

Si la conception juridique de la corruption, aujourd'hui dominante, ne convient pas à la pensée machiavélienne, c'est d'abord en fonction de l'orientation philosophique de celle-ci. Le républicanisme machiavélien peut être qualifié d' " hétérodoxe » par rapport à la tradition issue d'Aristote, notamment du fait que la conception machiavélienne de la vertu civique se déploie au sein d'une représentation de l'homme peu conforme à l'anthropologie qui, d'ordinaire, constitue la base des doctrines républicaines. D'une part, en effet, l'humain est pour Machiavel fondamentalement régi par un appétit qui se confond en lui avec l'impulsion vitale : par exemple, la tendance permanente qu'a un « Prince » d'entreprendre des conquêtes trahit le désir d'acquérir

9 Discours, III, $1,1$.

10 Nous avons analysé ce clivage dans une autre étude : cf. Thierry Ménissier, «L'usage civique de la notion de corruption selon le républicanisme ancien et moderne ", Anabases. Traditions et réception de l'Antiquité, $\mathrm{n}^{\circ} 6$ 2007, p. 83-98, accessible à l'URL : https://journals.openedition.org/anabases/3323 
qui, écrit-il, est « vraiment très naturel et ordinaire ${ }^{11}$, et une telle tendance caractérise, certes à des degrés divers, tous les individus de notre espèce. De l'autre, l'humain se trouve également animé par un désir de reconnaissance proprement inextinguible : il est mu par une pulsion fatalement déçue reposant sur l'asymétrie entre le désir de posséder (illimité) et la capacité à se satisfaire de ce qu'ils possèdent (très limitée) ${ }^{12}$. Le poids de ce que Machiavel appelle " l'ambition " apparaît ici fondamental, véritable « mauvaise graine » qui est la cause du mal enduré par l'homme dans l'histoire, pour reprendre les termes du Capitolo dell'Ambizione ${ }^{13}$. Avec ce thème de l'ambition, Machiavel signale qu'il existe naturellement dans le cœur des humains une puissance contraire au bien public.

Par suite, pour l'œuvre machiavélienne pourtant si éloignée de l'esprit du christianisme et de la thématique du péché originel, tout humain est non seulement potentiellement, mais également aisément corruptible. Comme l'explique le Discours, I, 42 (précisément intitulé «Combien les hommes peuvent facilement être corrompus »), les individus, même ceux qui sont correctement éduqués et plutôt enclins à se montrer honnêtes, peuvent être corrompus sans difficultés, c'est-à-dire qu'ils tendent à s'approprier privativement le bien public. C'est ce dont atteste, dans l'histoire romaine ainsi que la rapporte initialement Tite-Live, l'épisode de l'institution d'une magistrature spéciale, celle dite des « Dix » ou Decemvirs ${ }^{14}$. En dépit d'une volonté d'agir en faveur de la cause populaire, les acteurs de cette péripétie historique adoptèrent en effet des conduites défavorables au bien public et visant au bout du compte à satisfaire leur ambition personnelle, point qui, estime le Florentin, « rendra les législateurs des républiques ou des royaumes d'autant plus prompts à réfréner les appétits humains et à leur ôter tout espoir de pouvoir errer impunément $\gg^{15}$. Ce fâcheux penchant, ou mieux encore : cette pente fatale à la corruption sont encore plus sensibles dans l'épisode paradoxal dit de la Loi agraire $^{16}$. Ce terme recouvre un ensemble de lois, promues par Tiberius et Caius Gracchus, deux frères aristocrates favorables au peuple et qui occupèrent les fonction de tribuns, semblait permettre à ce dernier de s'approprier une partie de la terre publique, ce à quoi il aspirait légitiment compte tenu de la sujétion dans laquelle le tenait le système patricien des fermages et de la dette tandis que les conquêtes avaient étendu le domaine de Rome ${ }^{17}$. Or, explique Machiavel, et pour employer anachroniquement des termes contemporains, l'évolution sociale permise ou même simplement rendue pensable par la Loi agraire fut le début de la fin de la grandeur de Rome : elle mit un terme aux dispositions frugales indispensables pour maintenir

11 Voir Le Prince, III, $\S 40$ : «è cosa veramente molto naturale e ordinaria desiderare di acquistare ».

12 Voir Discours, I, 37, et II, avant-propos, les passages relatifs à la malacontentezza, terme qu'on peut traduire par «frustration » ou mieux encore par « ressentiment ». Un des points saillants de l'approche machiavélienne de la corruption de la vertu républicaine réside dans le fait que c'est souvent une telle disposition, et non l'appât franc et direct du gain, qui, chez les classes populaires ou intermédiaires, pousse à la captation indue du bien public et par-là subvertit l'ethos civique.

13 Voir le Capitolo de l'Ambition, traduction C. Bec, in Machiavel, Euvres, Paris, Robert Laffont, 1996, p. 10701075.

14 Rapporté par Tite-Live dans son Histoire de Rome depuis sa fondation (Ab Urbe condita), livre III, chapitre 35 ; analysé par Machiavel à plusieurs reprises, par exemple les Discours I, 35 et 40.

15 Machiavel, Discours, I, 42, p. 198-199.

16 Cf. Tite-Live, III, 1 ; Machiavel, Discours, I, 37.

17 Sur les réformes successivement entreprises par les frères Gracchus entre 133 et 121 avant J.-C., voir également, comme source ancienne, Plutarque, Vies parallèles, Les Gracques, trad. J.-M. Pailler, Paris, Gallimard, 2001, p. 1197-1536 ; et pour une mise en perspective historique, Claude Nicolet, Les Gracques. Crise agraire et révolution à Rome, Paris, Julliard, 1967. 
la cité puissante ${ }^{18}$. Le commentaire machiavélien de cette péripétie historique indique comment, en dépit de l'attachement de Machiavel à la cause du « peuple » opposé aux « grands », l'appétit naturel des humains est pour lui fauteur de corruption - il l'est à un point tel qu'il convient, si l'on veut sauver l'esprit républicain, de brider le désir du peuple.

\subsection{Une conception de la corruption plutôt civique que déontologique}

Politique, la conception machiavélienne gagne encore à être qualifiée comme plutôt civique que déontologique: ce qui importe au Florentin dans ses analyses des manières d'être du peuple, c'est la qualité de l'engagement des citoyens, plutôt que la rectitude de leurs manières d'être. On pourrait même imaginer que pour lui, cette rectitude peut être tout à fait réelle, et, pour autant, la République totalement corrompue. Explique ce paradoxe l'attention portée par le Florentin à des analyses qui se situent au plus près des phénomènes politiques, ainsi que nous voulons le montrer avec les quatre points suivants.

Premièrement, il y a risque de corruption des mœurs civiques lorsque «l'amour de la patrie » perd de sa vigueur. Dans une république, un des critères décisifs de la qualité de l'engagement réside en effet dans l'attachement fondateur des citoyens à la communauté civique. Le peuple est corrompu - ce qui signifie qu'il a perdu sa propre identité - lorsque ce marqueur éthique a disparu. Machiavel peut à cet égard être rangé (avec Aristote et Rousseau) parmi les auteurs que Benjamin Constant nommait «anciens» pour les distinguer des «modernes » (à savoir, de la tradition du libéralisme) ${ }^{19}$. De fait, le Florentin argumente dans maints passages sur la décadence des mœurs dont il est contemporain vis-à-vis de celles de l'Antiquité. En attestent par exemple les attaques en règle dans les Discours sur Tite-Live contre la religion chrétienne : les promoteurs d'une morale qui a consisté à « désarmer le ciel et efféminer le monde » sont dénoncés, sur le plan de la civilisation, comme les fauteurs d'une dégradation irrémédiable de la vertu civique ${ }^{20}$.

En poursuivant la mise en perspective historique rendue possible par la distinction cardinale entre Anciens et Modernes, on pourrait dire que la pensée du Florentin pourrait permettre d'évaluer la dégradation de l'éthos civique selon une double tendance : celle de l'essor de l'individualisme (analysé après Constant par Tocqueville), bien entendu, mais également celle l'affirmation d'affiliations communautaires antagoniques à la république, qu'elles soient de type pré-politiques (comme dans le cas du revivalisme religieux), ou de type post-politique (comme dans le cas de l'émergence de communautés socio-culturelles, numériques ou non, toujours «factieuses » en regard de la communauté politique, car fragmentant celle-ci). En somme, derrière le thème de la perte de «l'amour de la patrie », ce qui est en jeu, c'est la nécessité de maintenir ou même de réinventer une communauté civique, tâche fondamentale pour une pensée politique contemporaine. Un des paradoxes que permet de souligner la pensée machiavélienne est que c'est peut-être le triomphe de l'État, quand on le conçoit seulement comme une structure juridique régie par une gestion technocratique « dépersonnalisante », qui contribue à la perte de valeur de l'affect, ciment fondamental de la communauté civique.

18 «[... les républiques bien ordonnées doivent maintenir riche le trésor public et leurs citoyens pauvres...», Discours, I, 37, 1.

19 Voir Benjamin Constant, « De la liberté des Anciens comparée à celle des Modernes », in De la liberté chez les Modernes, textes choisis, présentés et annotés par M. Gauchet, Paris, L.G.F., 1980, p. 494-495.

20 Discours, II, 2, 2. 
Deuxièmement, c'est l'affaiblissement du désir d'égalité, et corrélativement ce qu'on pourrait nommer de manière anachronique la «privatisation des conduites » qui corrompent le peuple. Ce qui peut signifier plusieurs choses. D'abord, cela renvoie à la perte de ce que les Grecs avaient nommé isonomie (à savoir, le principe selon lequel la loi soit la même pour tous), lorsque la volonté de s'appliquer à soi-même la contrainte légale apparaît pour les citoyens à la fois moins forte et moins constante. Certains passages importants des Discours portant sur la difficulté extrême des «cités corrompues » (città corrotte) à demeurer libres, passages manifestement très travaillés par l'auteur ${ }^{21}$, mettent en exergue le fait que, lorsqu'un tel affaiblissement se produit, même les meilleures institutions et les lois spécialement établies pour contrer cette tendance peuvent s'avérer des obstacles et fragiliser l'action politique. Ensuite, cela renvoie à la secondarisation de la chose publique par rapport aux intérêts économiques. Dans son autre grand livre républicain, les Histoires florentines, tout particulièrement, le Secrétaire se livre à une enquête minutieuse sur la manière dont, dans l'histoire récente de sa propre cité, le bien public a été détourné au profit de l'intérêt personnel et clanique, et comment la puissance publique a été amoindrie avec l'essor de la richesse privée. $\mathrm{Au}$ fil de la narration de son histoire, l'antique et vénérable patrie du vivere politico se trouve confrontée à la réalité de la montée du commerce moderne, promu par les grandes familles florentines (y compris par les Médicis qui, revenus au pouvoir, ont commandé à Machiavel son livre sur l'histoire de la cité). Pour employer les termes qui composent le titre d'un ouvrage connu, les «passions » ont succédé aux «intérêts » en tant que ressort de la vie sociale et politique $^{22}$.

Toutefois, la thématique de l'égalité telle que Machiavel l'entend dans ses œuvres (equalità) ne doit pas être comprise dans l'optique de l'égalitarisme social (que le Secrétaire réfute implicitement dans un passage fameux des Histoires florentines ${ }^{23}$ ) ni même en fonction de la théorie moderne de l'égalité des individus du point de vue de leurs droits naturels, civils et civiques. Dans plusieurs textes importants, en regard de l'opposition entre les types de régimes qui se montrent soit favorable soit défavorable à l'égalité, certaines explications sont fournies par l'auteur, qui apparaissent explicites sur le plan de la stratégie politique bien qu'elles ne semblent guère précises sur celui de la conceptualité ${ }^{24}$. Les arguments fournis donnent à penser une idée d'égalité conçue dans l'esprit du système corporatiste qui régissait les relations entre «métiers » (arti) au sein de la cité florentine. Sans placer tous les acteurs sociaux sur le même plan, et sans leur accorder les mêmes prérogatives, ce système offrait à la plupart d'entre eux

21 Voir ici l'argumentation très tendue de Discours, I, 18, où l'analyse des difficultés de juguler la corruption civique se fonde sur une double thématique : la circularité des lois et des mœurs, d'une part (les mauvaises mœurs font des mauvaises lois qui engendrent de mauvaises mœurs), et la distinction entre institutions fondamentales et nouvelles lois créées ad hoc, de l'autre.

22 Voir Albert Hirschman, Les passions et les intérêts. Justifications politiques du capitalisme avant son apogée, trad. P. Andler, Paris, P.U.F., 1980.

23 Voir le passage où il traite de l'émeute des ouvriers de la laine, révoltés, les Ciompi, et de leurs revendications «égalitaristes »: Histoires florentines, livre III, chapitres 12-21: si Machiavel ne se prononce explicitement ni pour ni contre le contenu du discours très puissant qu'il met lui-même dans la bouche du personnage leader des rebelles (III, 13), la « libération » de la parole des ouvriers paraît contemporaine dans son texte d'une subtile évaluation négative de leur action. Cette dernière obéit en effet à une logique extrémiste apparemment appropriée à leur situation, mais qui se trouve en contradiction avec la survie et le renforcement de la cité en fonction de la longue durée - perspective qui constitue pourtant la visée implicite des Histoires florentines, et d'après laquelle sont évalués tant les gouvernements que les forces sociales qui se trouvent aux prises.

24 Voir Discours, I, 55, et Discours sur les choses de Florence après la mort de Laurent de Médicis le jeune (1520), trad. C. Bec in Machiavel, Euvres, op. cit., p. 79-91. 
les conditions d'une expression publique, sinon un certain pouvoir politique. Machiavel forge en quelque sorte une représentation idéal-typique de ce système, qui serait pertinente pour déterminer le meilleur régime pour sa cité. On pourrait même se risquer à proposer une idée plus précise du contenu de sa théorie de l'égalité en la référant, mutatis mutandis, à la manière dont Aristote dans l'Éthique à Nicomaque qualifie à partir de l'invention du concept de philia la relation de parité que peuvent entretenir des participants à l'échange social qui sont pourtant susceptibles d'être (très) inégaux dans leur statut naturel comme dans leurs prérogatives sociopolitiques. Si la relation qu'entretiennent le mari et la femme, ou le maître et le serviteur, est malgré tout basée sur une sorte d'égalité, c'est que, une fois comprise dans un cadre qui leur accorde les prérogatives correspondant à leur position effective, elle correspond à une relation sociale authentique et donne lieu à des échanges sincères ${ }^{25}$. Non que Machiavel revendique jamais une affiliation avec l'argumentation du Stagirite, mais celle-ci fournit un archétype intellectuel susceptible de schématiser la notion machiavélienne d' " equalità ». Dans le système politique issu d'une telle conception de l'égalité, chacun occupe la place publique que sa situation sociale lui fait espérer : il existe donc une parité politique entre tous, sans nier les différences de condition, de fortune et de statut.

Troisièmement, il convient de souligner un caractère original de la corruption du peuple tel que l'entend le Florentin, un caractère assez éloigné des habitudes mentales en tant qu'il se trouve lié au caractère hétérodoxe du républicanisme machiavélien. Au sein de ce dernier, se verrait altérée par la corruption toute république où font défaut les « tumultes » favorables à la préservation liberté civique. Loin de l'intuition ordinaire, l'esprit de consensus partagé constitue même l'indicateur d'une perte d'identité dangereuse, tandis que l'attention aux discordes internes représente le signe de la vitalité, dans la mesure où elle traduit la capacité à maintenir en éveil le désir d'autonomie. Cela est vrai pour l'ensemble de la république, à partir de l'histoire ancienne de Rome envisagée comme un modèle. Mais, concernant le peuple, et alors qu'il analyse l'histoire moderne de Florence, Machiavel suggère une comparable mise en garde à l'encontre de ce qui pourrait représenter pour le peuple la tentation conjuguée de l'hégémonie politique et de l'homogénéité sociale. La cité du Lys ne s'est nullement trouvée renforcée, mais fut bel et bien affaiblie lorsque le peuple a presque réussi à se débarrasser de ses adversaires les nobles : les dispositions actives de la cité se sont vues affaiblies sous l'effet d'un appauvrissement de l' " humeur » aristocratique (faite, est-il précisé, de virtù delle armi et de generosità di animo), créant un manque préjudiciable que l'«humeur » populaire est incapable de compenser par ses seules forces ${ }^{26}$.

Quatrièmement, sur le plan de l'organisation interne, il y a corruption lorsque la rétribution sociale des conduites ne fonctionne plus. Machiavel aborde la question des « républiques bien ordonnées » (republiche bene ordinate) en fonction de leur capacité à décerner aux citoyens récompenses et punitions avec la justesse appropriée ${ }^{27}$. Une telle analyse engage le problème abyssal de la rétribution sociale des conduites civiques (ainsi que celui, plus particulier, du rapport entre récompenses et punitions). Mais pour abyssal qu'il soit, ce problème n'en est pas moins cardinal pour le républicanisme. En effet, si ce problème apparaît

25 Cf. Aristote, Ethique à Nicomaque, VIII, 1, 1155 a 1 - IX, 2, 1165 a 35.

26 Voir Histoires florentines, III, 1, in Machiavel, Euvres, trad. C. Bec, op.cit., p. 751-752.

27 Discours, I, 24, § 1. 
crucial, c'est qu'ici se joue en quelque sorte la possibilité même d'une civilité politique, ce dont Machiavel a eu une conscience tout à fait claire ainsi qu'on peut le lire dans ce passage :

[...] Aucune république bien ordonnée n'effaça jamais les démérites de ses citoyens par leurs mérites ; mais, ayant établi des récompenses pour une action bonne et des peines pour une mauvaise, et ayant établi des récompenses pour une action bonne, s'il par la suite celui-ci agit mal, elle le châtie sans aucun égard pour ses actions bonnes. Et si ces institutions sont bien observées, une cité vit libre longtemps : autrement, elle ira toujours à sa perte rapidement. En effet, si un citoyen qui aurait accompli quelque action illustre pour la cité joint à la réputation que cela lui apporte l'audace et l'assurance de pouvoir faire, sans crainte de châtiment, alors il deviendra en peu de temps si insolent que toute vie civile se dissoudra [diventerà in brieve tempo tanto insolente che si risolverà ogni civiltà $]^{28}$.

Le principe de la vie civile risque d'être profondément affecté s'il règne en république un esprit d'impunité tel que les hommes excellents bénéficient pour cela même d'un passe-droit leur permettant de commettre des mauvaises actions. La confusion entre le licite et l'illicite, amplifiée par la réputation d'excellence qui masquerait le vice, sont susceptibles de produire des désastres sur la vie politique. Ici la thématique de la corruption renvoie au système de l'équilibre entre les mérites civiques des individus et leurs actions blâmables, et avec elle se joue la possibilité même d'une régulation éthique des mœurs, dans un texte qui manifeste, il convient de le reconnaître, une certaine ambition axiologique.

\section{Conclusion : des indicateurs de la corruption et de la nature du peuple}

$\mathrm{Au}$ final, quels sont les indicateurs permettant d'évaluer la vertu civique et qui permettent de juger si elle est ou non corrompue ? Il est remarquable que Machiavel propose en quelque sorte un système à double entrée. Pour le responsable politique, « prince » ou leader d'une république, le critère de la vertu tient dans sa capacité à mantenere lo stato, soit dans sa faculté à « conserver son état (ou son État) »; et, toutes choses naturelles étant appelées à se dégrader, l'indicateur de corruption réside dans la durée du maintien. Au niveau des simples citoyens, en dépit d'un facteur tel que l'avidité naturelle des humains, le degré de la corruption civique se décèle dans l'attention qu'ils portent à leur cité. Il tient, par suite, aux soins dont les citoyens entourent la société politique dont ils sont membres, et, mieux encore, à l'engagement dont ils font preuve en faveur du collectif. Le tout premier facteur de corruption de la cité réside donc dans l'indifférence des citoyens à l'égard de la chose publique ; et toute la question, pour éviter la corruption, est de savoir de quelle manière contrer cette dangereuse tendance. Comment réanimer sans cesse l'attention à la chose publique dans le cœur des hommes avides ? La société publique ne saurait durer sans le consentement actif du peuple à la participation civique; mais ce consentement n'est lui-même possible que grâce à l'exemplarité des responsables politiques.

Une autre remarque conclusive peut être faite, qui concerne la nature du peuple. Sujet de référence de la bonne politique en république, exprimant la puissance collective ou du collectif, ce dernier ne constitue pas pour autant aux yeux de Machiavel le principe impeccable

\footnotetext{
${ }^{28}$ Ibidem, $\S 2$.
} 
de la droiture politique. Au contraire, pour le Florentin, subtil observateur des pratiques politiques, l'action collective apparaît susceptible d'être errante, à savoir, irrésolue et mal orientée. D'autres penseurs républicains ont partagé un même souci de saisir le degré de maturité du peuple, ainsi Rousseau ${ }^{29}$. Le peuple, comme sujet collectif concret, est en effet parfois capable de ne pas vivre comme il devrait le faire pour se montrer conforme à la politeia qui lui correspond, à savoir, la forme de vie républicaine, dont les deux ressorts sont la participation des citoyens et l'engagement des représentants, et la règle, le respect de la valeur sacrée de la chose publique. Voilà pourquoi Machiavel est un auteur important : sur le plan politique, réfléchir aux événements vécus de l'histoire à l'aide de sa pensée transmet aux collectifs et à leurs leaders une précieuse disposition de vigilance vis-à-vis de leurs propres tendances. Et sur le plan philosophique, le lire permet de dépasser la tentation récurrente de postuler pour la notion de "peuple » un caractère substantiel. Le peuple n'est pas une réalité qui existe une fois pour toute, un sujet constitué. Sont du peuple les collectifs qui, faisant l'épreuve de leurs propres difficultés à se constituer et à durer, réussissent, en inventant des formes de subjectivation originales, à agir et à transformer l'histoire.

\section{Auteur}

Thierry Ménissier est professeur de philosophie politique à l'Université Grenoble Alpes et membre de l'Institut de Philosophie de Grenoble (IPhiG). Ses travaux, d'inspiration machiavélienne, portent sur la transformation des principes de l'éthique publique, sur le sens de l'innovation technologique et sociale, et sur l'éthique pertinente pour le rapport humainsmachines d'aujourd'hui. Il a notamment publié : Machiavel ou la politique du Centaure (Hermann, 2010); La Liberté des contemporains. Pourquoi il faut rénover la République (PUG, 2011) ; Machiavel, Ombres et lumières du politique (Ellipses, 2017) ; Philosophie de la corruption (Hermann, 2018); Innovations. Une enquête philosophique (Hermann, 2021).

IPhiG, Université Grenoble Alpes, Bâtiment ARSH, BP 47, Domaine Universitaire, 1281, avenue Centrale, 38400 Saint-Martin d'Hères

$\underline{\text { Thierry.menissier@univ-grenoble-alpes.fr }}$

29 Voir Jean-Jacques Rousseau, Du Contrat social, III, 8 : « Du peuple ». 